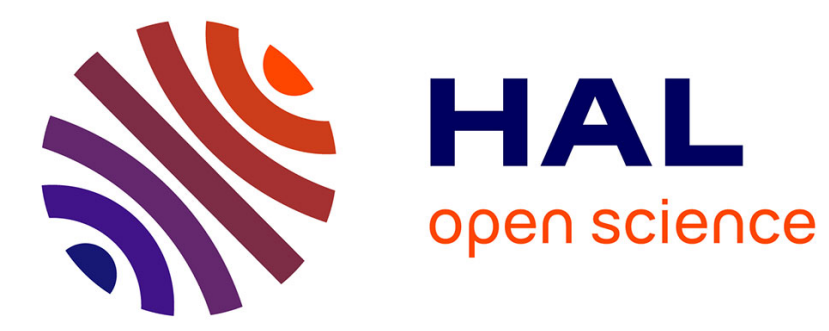

\title{
A Separable Instrumental Variable Method for Robot Identification
}

\author{
Alexandre Janot
}

\section{To cite this version:}

Alexandre Janot. A Separable Instrumental Variable Method for Robot Identification. ACC 2021, May 2021, NEW ORLEANS, United States. 10.23919/ACC50511.2021.9482821 . hal-03346290

\section{HAL Id: hal-03346290 \\ https://hal.science/hal-03346290}

Submitted on 16 Sep 2021

HAL is a multi-disciplinary open access archive for the deposit and dissemination of scientific research documents, whether they are published or not. The documents may come from teaching and research institutions in France or abroad, or from public or private research centers.
L'archive ouverte pluridisciplinaire HAL, est destinée au dépôt et à la diffusion de documents scientifiques de niveau recherche, publiés ou non, émanant des établissements d'enseignement et de recherche français ou étrangers, des laboratoires publics ou privés. 


\title{
A Separable Instrumental Variable Method for Robot Identification
}

\author{
A. Janot
}

\begin{abstract}
This paper addresses the topic of robot model identification. The standard identification method is based on the use of the inverse dynamic model (IDM) and the application of Least Squares (LS) estimation while the robot is tracking trajectories. Although this approach has been applied successfully to several industrial robots, the standard friction model is assumed to be described by a linear relationship in the viscous and Coulomb coefficients and the consistency of LS estimates is not secured when the system is identified in closed loop. In this paper, a Separable Instrumental Variable (SIV) approach is proposed that corrects these limitations. This SIV method consists of twosteps. In the first one, a Nonlinear LS estimation is combined with the IDM to identify the nature of friction effect, which is potentially nonlinear. In a second step, an iterative linear IV method is performed in order to obtain the estimates of the inertia and gravity parameters. The experimental results obtained on the 6 degrees-of-freedom TX40 robot show the effectiveness of this new approach.
\end{abstract}

\section{INTRODUCTION}

This paper deals with robot identification which has a long history, see [1]-[6] and the references therein, and is witnessing a renew, see [7]-[11] among others. The standard approach to modelling robots is based on an inverse dynamic identification model combined with leastsquares estimation. This method, referred to as the Inverse Dynamic Identification Model with Least Squares (IDIMLS), has been successfully applied to several prototypes and industrial robots, see e.g. [1]-[6]. Good results can be obtained provided a well-tuned derivative bandpass filtering of the joint position measurements is used to calculate the joint velocities and accelerations. However, because robots are identified during closed-loop operation, the user can never be sure that the IDIM-LS estimates are consistent, even if a tailor-made data filtering is used [6], [12]-[14]. Instrumental Variable (IV) estimation provides an alternative approach to this problem and, in [6], an IV method suitable for robot identification, called IDIM-IV, has been developed and validated experimentally. It has been shown that this method is robust in the presence of measurement noise, and the instruments can be evaluated using a revised Durbin-Wu-Hausman test (DWH-test) statistic, [15]. However, the friction model is described by a linear relationship in the viscous and Coulomb coefficients.

Friction modelling and identification have a long history and this topic is so vast and rich that it is impossible to cite all the relevant works. The interested reader can refer to the following survey [16] and the references therein. Friction models can be divided into two classes: static models that depend only on the current

A. Janot is with French Aerospace Lab, 6 Chemin de la Vauve aux Granges, 91120 Palaiseau, France (Phone: +33-1-80-38-65-76; e-mail: Alexandre.Janot@onera.fr ). velocity and dynamic models that are more general. Note that models which are temperature-dependent have been considered, [7] and [17]-[18]. As regards identification methods, nonlinear estimation techniques are popular since the friction models are mostly nonlinear, see e.g. [17]- [18] amongst others.

If we consider static friction models, an interesting approach is the exploitation of the Separable LS approach (SLS), [19]-[20]. The SLS method assumes that the dynamic model can be split into two independent parts: one linear to a set of parameters and another one nonlinear to another set of parameters. Like the parts, the two sets are assumed to be independent. Regarding the estimation process, SLS consists of two steps: in the first one, the nonlinear parameters are estimated thanks to a non-linear LS algorithm (NLS), and in the second, we use the linear LS. Then, an iterative process is run to estimate the dynamic parameters. According to [19], separating the NLS problem improves the convergence of the algorithm.

Surprisingly, the SLS method has not received much attention in robotics, although its potential for use in this context was reported some years ago, [21]. This may be due to the fact that the researchers and engineers prefer to run a whole NLS method to estimate the dynamic parameters. However, because the SLS method makes use of the linear LS approach, it lacks robustness against a correlation between the observation matrix and the vector of errors. To overcome this drawback, an extension of the IDIM-IV method could be applied, as suggested years ago in [6].

In this paper, we focus on the identification of static friction models without any other prior assumptions about the nature of the nonlinearities. The contribution of this paper is two-fold. First, a Separable IV (SIV) approach making use of the principles of the SLS and IDIM-IV methods, termed the IDIM-SIV method, is introduced and experimentally validated on the TX40 robot. It is shown that IDIM-SIV yields an accurate estimation of non-linear friction characteristics. Second, the inertial parameters of the robot are identified by the IDIM-IV method based on the friction characteristics estimated in the first step. The experimental results show that IDIM-SIV is more robust against noises than SLS, and outperforms a classical closed-loop output-error (CLOE) method.

The remaining sections of the paper are organized as follows: Section II reviews the standard IDIM-LS method; Section III introduces the new IDIM-SIV approach; Section IV is devoted to the experimental results; and Section V summarises our conclusions. 


\section{THE STANDARD IDIM-LS METHOD FOR ROBOT IDENTIFICATION}

\section{A. Inverse dynamic model of robots}

The inverse dynamic model (IDM) of robots with $n$ moving links calculates the $(n \times 1)$ joint torques vector $\boldsymbol{\tau}_{i d m}$ as a function of generalized coordinates and their derivatives, [22]:

$$
\boldsymbol{\tau}_{i d m}=\mathbf{M}(\mathbf{q}) \ddot{\mathbf{q}}+\boldsymbol{\tau}_{c o r}(\mathbf{q}, \dot{\mathbf{q}})+\boldsymbol{\tau}_{g}(\mathbf{q})+\boldsymbol{\tau}_{f},
$$

where $\mathbf{q}, \dot{\mathbf{q}}$ and $\ddot{\mathbf{q}}$ are respectively the $(n \times 1)$ vectors of generalized joint positions, velocities and accelerations; $\mathbf{M}(\mathbf{q})$ is the $(n \times n)$ inertia matrix; $\boldsymbol{\tau}_{c o r}(\mathbf{q}, \dot{\mathbf{q}})$ is the $(n \times 1)$ vector of centrifugal and Coriolis torques; $\boldsymbol{\tau}_{g}(\mathbf{q})$ is the $(n \times 1)$ vector of gravitational torques; and $\boldsymbol{\tau}_{f}$ is the $(n \times 1)$ vector of friction torques. In robotics, it is convenient to introduce $\mathbf{N}(\mathbf{q}, \dot{\mathbf{q}})$, the $(n \times 1)$ vector that encompasses the centrifugal, Coriolis, gravitational and friction torques with $\mathbf{N}(\mathbf{q}, \dot{\mathbf{q}})=\boldsymbol{\tau}_{\text {cor }}(\mathbf{q}, \dot{\mathbf{q}})+\boldsymbol{\tau}_{g}(\mathbf{q})+\boldsymbol{\tau}_{f}$. The IDM (1) can be written, therefore, as $\boldsymbol{\tau}_{i d m}=\mathbf{M}(\mathbf{q}) \ddot{\mathbf{q}}+\mathbf{N}(\mathbf{q}, \dot{\mathbf{q}})$.

The 11 inertial parameters of a link $j$ are, see [22]: $X X_{j}, X Y_{j}, X Z_{j}, Y Y_{j}, Y Z_{j}$ and $Z Z_{j}$, the six components of the inertia matrix of link $j$ at the origin of frame $j ; M X_{j}, M Y_{j}$ and $M Z_{j}$, the components of the first moment of link $j ; M_{j}$ is the mass of link $j$; and $I a_{j}$ a total inertia moment for the rotor and gears of actuator $j$. The set of identifiable inertial parameters, called set of base parameters, has to be determined because all of the inertial dynamical parameters are not identifiable: some have no influence on the IDM while others are regrouped via linear relations, see [22]. By denoting $\boldsymbol{\beta}_{L}$ as the $\left(b_{L} \times 1\right)$ vector of identifiable inertial parameters with $b_{L}$ as the minimum number of identifiable inertial parameters from which the IDM can be calculated, the modified Denavit and Hartenberg (DHM) notation allows for the formulation of the following IDM which is linear in relation to $\boldsymbol{\beta}_{L}$,

$$
\boldsymbol{\tau}_{i d m}=\mathbf{I D M}_{L}(\mathbf{q}, \dot{\mathbf{q}}, \ddot{\mathbf{q}}) \boldsymbol{\beta}_{L}+\boldsymbol{\tau}_{f}\left(\boldsymbol{\beta}_{f}\right),
$$

$\operatorname{IDM}_{L}(\mathbf{q}, \dot{\mathbf{q}}, \ddot{\mathbf{q}})$ is the $\left(n \times b_{L}\right)$ matrix of basis functions associated with $\boldsymbol{\beta}_{L} ; \boldsymbol{\beta}_{f}$ is the $\left(b_{f} \times 1\right)$ vector of parameters of friction torques; and $b_{f}$ is the number of parameters required to model the friction characteristics.

Assuming that the joint $j$ friction torque, $\tau_{f_{j}}$, is modelled by the standard linear relationship

$\tau_{f_{j}}=F v_{j} \dot{q}_{j}+F c_{j} \operatorname{sign}\left(\dot{q}_{j}\right)$, where $F v_{j}$ and $F c_{j}$ the viscous and Coulomb friction parameters of joint $j$, the IDM (2) is linear in a set of identifiable parameters, $\boldsymbol{\beta}$, i.e.

$\boldsymbol{\tau}_{i d m}=\operatorname{IDM}(\mathbf{q}, \dot{\mathbf{q}}, \ddot{\mathbf{q}}) \boldsymbol{\beta}$,

where $\operatorname{IDM}(\mathbf{q}, \dot{\mathbf{q}}, \ddot{\mathbf{q}})$ is the $(n \times b)$ matrix of basis functions of the bodies dynamics; $\boldsymbol{\beta}=\left[\begin{array}{ll}\boldsymbol{\beta}_{L}^{T} & \boldsymbol{\beta}_{f}^{T}\end{array}\right]^{T}$ is the $(b \times 1)$ vector of identifiable parameters; and $b=b_{L}+b_{f}$ is the number of identifiable parameters with $b_{f}=2 \cdot n$. Because of errors, the $(n \times 1)$ vector of actual joint torques, $\boldsymbol{\tau}$, differs from $\boldsymbol{\tau}_{i d m}$ by an error $\mathbf{e}_{i d m}$. We obtain the Inverse Dynamic Identification Model (IDIM) given by

$$
\boldsymbol{\tau}=\operatorname{IDM}(\mathbf{q}, \dot{\mathbf{q}}, \ddot{\mathbf{q}}) \boldsymbol{\beta}+\mathbf{e}_{i d m} .
$$

\section{B. Data acquisition and data filtering}

The data available from robot controllers are the measurements of $\mathbf{q}$ and the $(n \times 1)$ control signal vector $\mathbf{v}_{\tau}$. The joint $j$ control signal, $v_{\tau_{j}}$, is connected with the joint $j$ torque, $\tau_{i d m_{j}}$, by

$\tau_{i d m_{j}}=g_{\tau_{j}} v_{\tau_{j}}$

where $g_{\tau_{j}}$ is the joint $j$ drive gain, which has an a priori value provided by the manufacturers that can be reidentified using special tests, [23].

\section{IDIM-LS estimates}

In robotics, we are considering a closed-loop situation and this requires a special approach to identification. Usually, a pragmatic approach based on an efficient tailormade method of data filtering is used, see e.g. [5]. The IDIM given by (5) is sampled at a measurement frequency $f_{m}$ while the robot is tracking reference trajectories $\left(\mathbf{q}_{r}, \dot{\mathbf{q}}_{r}, \ddot{\mathbf{q}}_{r}\right)$. Then, after data sampling, the following over-determined system is obtained,

$\mathbf{y}(\boldsymbol{\tau})=\mathbf{X}(\hat{\mathbf{q}}, \hat{\dot{\mathbf{q}}}, \hat{\mathbf{q}}) \boldsymbol{\beta}+\boldsymbol{\varepsilon}$,

where $\mathbf{y}(\boldsymbol{\tau})$ is the $(r \times 1)$ measurements vector built from actual torques $\boldsymbol{\tau} ; \mathbf{X}(\hat{\mathbf{q}}, \hat{\dot{\mathbf{q}}}, \hat{\mathbf{q}})$ is the $(r \times b)$ observation matrix from $\operatorname{IDM}(\hat{\mathbf{q}}, \hat{\dot{\mathbf{q}}}, \hat{\mathbf{q}}) ; \boldsymbol{\varepsilon}$ is the $(r \times 1)$ sampled vector of $\mathbf{e}_{i d m} ; r=n_{e} \cdot n$ is the number of rows in (7); and $n_{e}$ is the number of samples collected for each joint $j$.

Based on the usual assumptions of linear LS estimation, the IDIM-LS estimates and their associated covariance matrix are given by $\hat{\boldsymbol{\beta}}_{L S}=\left(\mathbf{X}^{T} \mathbf{X}\right)^{-1} \mathbf{X}^{T} \mathbf{y}(\boldsymbol{\tau})$, $\boldsymbol{\Sigma}_{L S}=\hat{\sigma}_{\varepsilon}^{2}\left(\mathbf{X}^{T} \mathbf{X}\right)^{-1}$, with $\quad \hat{\sigma}_{\varepsilon}^{2}=\left\|\mathbf{y}(\boldsymbol{\tau})-\mathbf{X} \hat{\boldsymbol{\beta}}_{L S}\right\| /(r-b)$. 
$\hat{\sigma}_{\hat{\beta}_{L S}(i)}^{2}=\boldsymbol{\Sigma}_{L S}(i, i)$ is the i-th diagonal coefficient of $\boldsymbol{\Sigma}_{L S}$.

\section{Limitations of the IDIM-LS method}

Despite the fact the standard IDIM-LS method has been validated on many robots and prototypes, it has three main limitations. First, the friction is assumed to be modelled by the linear relationship in (3). If the friction phenomenon exhibits additional non-linear effects, IDIM-LS is no longer suitable. Second, robots are identified in closedloop because they are effectively double-integrator systems that cannot be identified in open-loop. It is well known that, in this situation, the LS estimates are asymptotically biased because $\mathbf{X}(\hat{\mathbf{q}}, \hat{\mathbf{q}}, \hat{\mathbf{q}})$ is correlated with $\boldsymbol{\varepsilon},[12]-[14]$, and [24]. Finally, the direct dynamic model (DDM) given by

$\ddot{\mathbf{q}}=\mathbf{M}^{-1}(\mathbf{q})\left(\boldsymbol{\tau}_{i d m}-\mathbf{N}(\mathbf{q}, \dot{\mathbf{q}})\right)$,

is validated a posteriori.

\section{A SEPARABLE IV APPROACH}

The IDIM-SIV makes use of the principles of SLS. In the first step, we estimate the graphical nature of the frictional effect in each joint to estimate $\boldsymbol{\beta}_{f}$. In the second step, we estimate $\boldsymbol{\beta}_{L}$. This defines an iterative algorithm executed until convergence.

\section{A. First step: estimating the graphical nature of frictions}

To estimate the graphical nature of the frictional effect, we excite each link while the others are maintained at their steady-states. This approach is common in robotics: see e.g. [10], [16]-[18]. When only the link $j$ is moving, while the other links stay at their steady-states, its IDM is given by

$\tau_{j}=Z Z_{j} \ddot{q}_{j}+\tau_{g_{j}}+\tau_{f_{j}}\left(\dot{q}_{j}\right)$,

where $\tau_{j}$ is the actual joint $j$ torque; $\tau_{g_{j}}$ is the joint $j$ gravity torque given by $\tau_{g_{j}}=-g M X_{j} \cos \left(q_{j}\right)+g M Y_{j} \sin \left(q_{j}\right) ; g$ is the gravity constant with $g=9.81$; and $\tau_{f_{j}}\left(\dot{q}_{j}\right)$ is the velocitydependent joint $j$ friction torque, whose the form is a priori unknown to the user. We can extract the graphical nature of the frictional effect with the following relation

$\hat{\tau}_{f_{j}}^{0}\left(\dot{q}_{j}\right)=\tau_{j}-\widehat{Z Z}_{j}^{0} \ddot{q}_{j}-\hat{\tau}_{g_{j}}^{0}$,

with $\hat{\tau}_{g_{j}}^{0}=-g \widehat{M X}_{j}^{0} \cos \left(q_{j}\right)+g \widehat{M Y}_{j}^{0} \sin \left(q_{j}\right)$, where $\widehat{Z Z}_{j}^{0}$, $\widehat{M X}_{j}^{0}$, and $\widehat{M Y}_{j}^{0}$ are the Computer-Aided-Design (CAD) values of $Z Z_{j}, M X_{j}$, and $M Y_{j}$, respectively. Then, by plotting $\hat{\tau}_{f_{j}}^{0}\left(\dot{q}_{j}\right)$ with respect to $\dot{q}_{j}$, we obtain the graphical nature of the joint $j$ frictional effect, we can select an appropriate friction model, and define $\boldsymbol{\beta}_{f_{j}}$, the vector of parameters describing the joint $j$ friction model. Finally, by applying a NLS method, one obtains an initial estimate of $\boldsymbol{\beta}_{f_{j}}$, denoted $\hat{\boldsymbol{\beta}}_{f_{j}}^{0}$. By assuming that the joint $j$ friction model is time-invariant so that the structure $\hat{\tau}_{f_{j}}^{k}\left(\dot{q}_{j}\right)$, the estimate of $\tau_{f_{j}}\left(\dot{q}_{j}\right)$ at iteration $k$, involves the same parameters as those in $\hat{\tau}_{f_{j}}^{0}\left(\dot{q}_{j}\right)$, we can estimate $\boldsymbol{\beta}_{L}$ at any iteration $k$.

\section{B. Second step: estimating $\boldsymbol{\beta}_{L}$}

At iteration $k$, let $\hat{\tau}_{f_{j}}^{k}$ be the joint $j$ friction characteristics identified in the first step, $\left(\hat{\boldsymbol{\tau}}_{f}^{k}\right)^{T}=\left[\begin{array}{lll}\hat{\tau}_{f_{1}}^{k} & \cdots & \hat{\tau}_{f_{n}}^{k}\end{array}\right]^{T}$ the $(n \times 1)$ vector of the identified friction characteristics, $\hat{\boldsymbol{\tau}}_{f-\text { samp }}^{k}$ the $(r \times 1)$ sampled vector of $\hat{\boldsymbol{\tau}}_{f}^{k}, \quad$ and $\left.\left(\begin{array}{lll}\hat{\boldsymbol{\beta}}_{f}^{k}\end{array}\right)^{T}=\left[\begin{array}{lll}\left(\hat{\boldsymbol{\beta}}_{f_{1}}^{k}\right)^{T} & \cdots & \left(\hat{\boldsymbol{\beta}}_{f_{n}}^{k}\right.\end{array}\right)^{T}\right]^{T}$ the vector of friction parameters. Assuming that the controller is known to the user, it is possible to simulate the DDM (8) with $\hat{\boldsymbol{\beta}}_{L_{N V}}^{k-1}$, the IV estimate of $\boldsymbol{\beta}_{L}$ at iteration $k-1$, and $\hat{\boldsymbol{\tau}}_{f}^{k}$, in order to get the $(n \times 1)$ vector of simulated joint accelerations given by

$\ddot{\mathbf{q}}_{S}=\mathbf{M}^{-1}\left(\mathbf{q}_{S}, \hat{\boldsymbol{\beta}}_{L_{V}}^{k-1}\right)\left(\boldsymbol{\tau}_{i d m_{S}}-\mathbf{N}\left(\mathbf{q}_{S}, \dot{\mathbf{q}}_{S}, \hat{\boldsymbol{\beta}}_{L_{V V}}^{k-1}, \hat{\boldsymbol{\beta}}_{f}^{k}\right)\right)$,

where $\quad \mathbf{N}\left(\mathbf{q}_{S}, \dot{\mathbf{q}}_{S}, \hat{\boldsymbol{\beta}}_{L_{N}}^{k-1}, \hat{\boldsymbol{\beta}}_{f}^{k}\right)=\boldsymbol{\tau}_{c o r}\left(\mathbf{q}_{S}, \dot{\mathbf{q}}_{S}, \hat{\boldsymbol{\beta}}_{L_{N}}^{k-1}\right)+$ $\boldsymbol{\tau}_{g}\left(\mathbf{q}_{S}, \hat{\boldsymbol{\beta}}_{L_{I V}}^{k-1}\right)+\hat{\boldsymbol{\tau}}_{f}^{k}\left(\mathbf{q}_{S}, \hat{\boldsymbol{\beta}}_{f}^{k}\right) ; \boldsymbol{\tau}_{i d m_{S}}$ is the $(n \times 1)$ vector of simulated joint torques whose the $\mathrm{j}$-th element is given by $\tau_{i d m_{s_{j}}}=C_{j}(s)\left(q_{r_{j}}-q_{S_{j}}\right)$; and $\mathbf{q}_{S}, \quad \dot{\mathbf{q}}_{S}$ are the $(n \times 1)$ vectors of the simulated joint positions and velocities obtained by integration of (11), respectively. In this case, at iteration $k$, the instrumental matrix is constructed as follows, [6],

$\mathbf{Z}_{L}^{k}=\mathbf{X}_{L}\left(\mathbf{q}_{S}, \dot{\mathbf{q}}_{S}, \ddot{\mathbf{q}}_{S}, \hat{\boldsymbol{\beta}}_{L_{N V}}^{k-1}\right)$,

where $\mathbf{X}_{L}\left(\mathbf{q}_{S}, \dot{\mathbf{q}}_{S}, \ddot{\mathbf{q}}_{S}, \hat{\boldsymbol{\beta}}_{L_{V}}^{k-1}\right)$ is the $\left(r \times b_{L}\right)$ observation matrix built from the sampling of $\operatorname{IDM}_{L}\left(\mathbf{q}_{S}, \dot{\mathbf{q}}_{S}, \ddot{\mathbf{q}}_{S}\right)$ and with $\hat{\boldsymbol{\beta}}_{L_{I V}}^{k-1}$. Let $\tilde{\mathbf{y}}_{S I V}^{k}=\mathbf{y}(\boldsymbol{\tau})-\hat{\boldsymbol{\tau}}_{f-\text { samp }}^{k}$ be the $(r \times 1)$ vector of measurements, where $\hat{\boldsymbol{\tau}}_{f-\text { samp }}^{k}$ has been removed from $\mathbf{y}(\boldsymbol{\tau})$ in order to keep the linear part of the IDM. The following over-determined system is obtained

$\tilde{\mathbf{y}}_{S I V}^{k}=\mathbf{X}_{L}(\hat{\mathbf{q}}, \hat{\dot{\mathbf{q}}}, \hat{\dot{\mathbf{q}}}) \boldsymbol{\beta}_{L}+\boldsymbol{\varepsilon}_{L}$,

where $\mathbf{X}_{L}(\hat{\mathbf{q}}, \hat{\dot{\mathbf{q}}}, \hat{\ddot{\mathbf{q}}})$ is the $\left(r \times b_{L}\right)$ observation matrix built 
from the sampling of $\operatorname{IDM}_{L}(\hat{\mathbf{q}}, \hat{\dot{\mathbf{q}}}, \hat{\overrightarrow{\mathbf{q}}}) ; \boldsymbol{\varepsilon}_{L}$ is the $(r \times 1)$ vector of error terms. The IV estimates are given by

$\hat{\boldsymbol{\beta}}_{L_{V}}^{k}=\left(\left(\mathbf{Z}_{L}^{k}\right)^{T} \mathbf{X}_{L}\right)^{-1}\left(\mathbf{Z}_{L}^{k}\right)^{T} \tilde{\mathbf{y}}_{S I V}^{k}$

$\mathbf{Z}_{L}^{k}$ being constructed with (12).

C. Convergence

With $\hat{\boldsymbol{\beta}}_{L_{I V}}^{k}$, we estimate $\hat{\boldsymbol{\beta}}_{f_{j}}^{k+1}$ by running a NLS method with $\hat{\tau}_{f_{j}}^{k+1}\left(\dot{q}_{j}\right)=\tau_{j}-\widehat{Z Z}_{j}^{k} \ddot{q}_{j}-\hat{\tau}_{g_{j}}^{k}$ where $\widehat{Z Z}_{j}^{k}$ and $\hat{\tau}_{g_{j}}^{k}$ are updated according to $\hat{\boldsymbol{\beta}}_{L_{V}}^{k}$. Then, we use $\hat{\boldsymbol{\beta}}_{f_{j}}^{k+1}$ to estimate $\hat{\boldsymbol{\beta}}_{L_{I V}}^{k+1}$, and so on.

Let $\hat{\boldsymbol{\beta}}_{S I V}=\left[\begin{array}{ll}\hat{\boldsymbol{\beta}}_{L_{V}}^{T} & \hat{\boldsymbol{\beta}}_{f}^{T}\end{array}\right]^{T}$ and $\mathbf{Z}_{L}$ be the SIV estimates obtained and instrumental matrix at the final iteration, respectively. Let $\boldsymbol{\varepsilon}_{I V}$ be the SIV error given by $\boldsymbol{\varepsilon}_{S I V}=\mathbf{y}(\boldsymbol{\tau})-\mathbf{Z}_{L}\left(\mathbf{q}_{S}, \dot{\mathbf{q}}_{S}, \ddot{\mathbf{q}}_{S}\right) \hat{\boldsymbol{\beta}}_{L_{I V}}-\boldsymbol{\tau}_{f}\left(\hat{\boldsymbol{\beta}}_{f}\right)$.

IDIM-SIV is iterated until $\left\|\boldsymbol{\varepsilon}_{S I V}^{k+1}\right\|-\left\|\boldsymbol{\varepsilon}_{S I V}^{k}\right\| /\left\|\boldsymbol{\varepsilon}_{S I V}^{k}\right\| \leq$ tol $_{1}$ and $\max _{i=1, \ldots, b}\left|\left(\hat{\boldsymbol{\beta}}_{S I V}^{k+1}(i)-\hat{\boldsymbol{\beta}}_{S I V}^{k}(i)\right) / \hat{\boldsymbol{\beta}}_{S I V}^{k}(i)\right| \leq t o l_{2}$ where $\left\|\boldsymbol{\varepsilon}_{S I V}^{k}\right\|$ is the norm of $\boldsymbol{\varepsilon}_{I V}$ at iteration $k$; and $\hat{\boldsymbol{\beta}}_{S I V}^{k}(i)$ is the i-th component of $\hat{\boldsymbol{\beta}}_{S I V}^{k}$ at iteration $k$. Parameters tol $_{1}$ and $\mathrm{tol}_{2}$ are values ideally chosen to get a good compromise between accuracy and the number of iterations needed to converge. A good compromise is to choose tol $_{1}$ and tol $_{2}$ between $2.5 \%$ and $5.0 \%$. $\hat{\boldsymbol{\beta}}_{L_{V}}^{k}$ is initialized with the CAD values of $\boldsymbol{\beta}_{L}$. The covariance matrices of $\hat{\boldsymbol{\beta}}_{L_{I V}}$ and $\hat{\boldsymbol{\beta}}_{f}$ are calculated according to standard rules, see e.g. [24] and section II.C. The relative error is given by $\% e_{I D I M-S I V}=100 \cdot\left\|\boldsymbol{\varepsilon}_{S I V}\right\| /\|\mathbf{y}(\boldsymbol{\tau})\|$.

Because this IV approach combines the SLS and the IDIM-IV methods, it is called IDIM-SIV.

\section{EXPERIMENTAL IDENTIFICATION RESULTS}

\section{A. Presentation of the TX40 Robot}

The TX40 robot has a serial structure with six rotational joints. The columns of $\operatorname{IDM}_{L}(\mathbf{q}, \dot{\mathbf{q}}, \ddot{\mathbf{q}})$ and the components of $\mathbf{M}(\mathbf{q})$ and $\mathbf{N}(\mathbf{q}, \dot{\mathbf{q}})$ are calculated with the Newton-Euler recursive algorithm implemented in the SYMORO+ software, [22]. The TX40 robot has 60 base dynamic parameters calculated with SYMORO+.

In order to excite the friction and inertial parameters, the exciting trajectories $\left(\mathbf{q}_{r}, \dot{\mathbf{q}}_{r}, \ddot{\mathbf{q}}_{r}\right)$ are designed so that $\dot{\mathbf{q}}_{r}$ are squares and $\ddot{\mathbf{q}}_{r}$ are trapezoidal, with various magnitudes. By combining these trajectories, one obtains $\operatorname{cond}(\mathbf{X})=200$, and $\left(\mathbf{q}_{r}, \dot{\mathbf{q}}_{r}, \ddot{\mathbf{q}}_{r}\right)$ that sufficiently excite the system and allow for good estimation of the base parameters, [25].

The TX40 robot is PID-controlled. For the three first joints, we have ${ }^{d} \omega_{n_{j}}=10 \mathrm{~Hz}$ and, for the last three joints, we have ${ }^{d} \omega_{n_{j}}=20 \mathrm{~Hz}$. For the six joints, the desired damping is set to ${ }^{d} \zeta_{j}=1.0$ to avoid overshoots. Finally, data are stored with a measurement frequency $f_{m}=1 \mathrm{kHz}$.

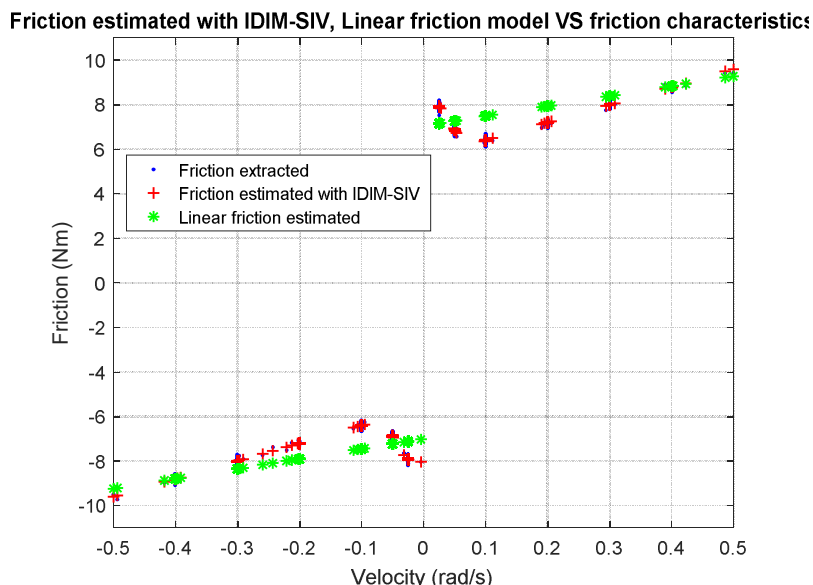

Fig. 1. The extracted friction characteristics for the second joint compared with the SIV-estimated friction shape and the standard linear friction model.

\section{B. Identification of the TX40 robot friction characteristics}

The estimate of the joint position, $\hat{\mathbf{q}}$, and its two time derivatives, $(\hat{\dot{\mathbf{q}}}, \hat{\hat{\mathbf{q}}})$, are calculated by an optimal filtering algorithm based on recursive fixed interval smoothing using the functions "irwsmopt" and "irwsm" of the CAPTAIN toolbox, [14].

The friction characteristics identified by the IDIM-SIV method for the second link are plotted in Fig. 1, which also shows the linear friction model estimated by the standard IDIM-LS method and the extracted shape. Similar results are obtained with the other joints and they are not presented here. The results obtained with the fifth and sixth joints are not reported here because the shapes captured by the IDIM-SIV method show that the usual linear friction model is sufficient in these cases.

According to the estimation results in Fig. 1, the friction characteristics represent a Stribeck effect so that, for the first four joints, $\tau_{f_{j}}\left(\dot{q}_{j}\right)$ can be modeled as

$$
\begin{array}{r}
\tau_{f_{j}}\left(\dot{q}_{j}\right)=F v_{j} \dot{q}_{j}+F c_{j} \operatorname{sign}\left(\dot{q}_{j}\right)+\ldots \\
\left(F s_{j}-F c_{j}\right) \exp \left(-\left(\frac{\left|\dot{q}_{j}\right|}{V s_{j}}\right)\right),
\end{array}
$$

where $F s_{j}$ is the joint $j$ stiction coefficient; $V s_{j}$ is the joint $j$ Stribeck velocity; and $\delta_{j}$ is the joint power coefficient that usually lies between 0.5 and 3.0. The parameters $F v_{j}, F c_{j}, F s_{j}, V s_{j}$ and $\delta_{j}$ are estimated by 
a standard nonlinear least-squares optimization algorithm, e.g. the "Isqnonlin" MATLAB function. The final estimates are regrouped in TABLE 1 and clearly emphasize the fact that the Stribeck effect is significant for the first three joints. Indeed, $F s_{j}$ differs significantly from $F c_{j}$.

\section{Identification of the inertia and gravity parameters of} the TX40 robot with the IDIM-IV method

As we have seen, the IDIM-IV method requires the synthesis of the matrix $\mathbf{X}_{L}(\hat{\mathbf{q}}, \hat{\dot{\mathbf{q}}}, \hat{\overrightarrow{\mathbf{q}}})$ and, given the use of IV-based estimation, there is no need for prior data filtering. The IDM-SIV method is initialized with CAD values for the inertia and gravity parameters while the friction parameters of joints 5 and 6 are initialized at 0 . To avoid the DDM simulation, the instrumental matrix is constructed with (12). Some base parameters have a poor contribution to the dynamics because they have small values and so can be omitted in order to simplify the dynamic models without affecting their accuracy. These are omitted after the application of the $\mathrm{F}$ test as explained in [6].

The IDM-SIV approach needs only 3 iterations to converge, and its estimates are given in TABLE 1. The relative error $\% e_{I D I M-S I V}$ is $5.3 \%$. The construction of $\mathbf{Z}_{L}$ has been validated by running the statistic presented in [15]. The instruments are valid and the IDIM-SIV estimates can be considered as statistically consistent.

\section{Comparison with other methods}

Now, the IDIM-SIV approach is compared with both the standard CLOE and SLS techniques.

Concerning the CLOE method, we recall that it minimizes the following criterion [5] and [6],

$J(\boldsymbol{\beta})=\frac{1}{2}\left\|\mathbf{q}_{S}-\mathbf{q}\right\|^{2}=\frac{1}{2}\left(\mathbf{q}_{S}-\mathbf{q}\right)^{T}\left(\mathbf{q}_{S}-\mathbf{q}\right)$,

where $\mathbf{q}_{S}$ is the simulated joint position which is the solution of the differential equation given by the DDM (8) assuming that both the actual and the simulated systems have the same input [5] and [6]. The minimization of $J(\boldsymbol{\beta})$ being a NLS problem, the estimates can be computed using algorithms such as the gradient method or Newton methods available in the "lsqnonlin" MATLAB function, see [26].

As regards the SLS approach, we recall that we the IDM given by (2), and $\boldsymbol{\beta}_{L}$ and $\boldsymbol{\beta}_{f}$ are estimated by making use of an iterative algorithm that involves a linear LS estimation of (13) for $\boldsymbol{\beta}_{L}$ i.e.

$\hat{\boldsymbol{\beta}}_{L_{L S}}^{k}=\left(\mathbf{X}_{L}^{T} \mathbf{X}_{L}\right)^{-1} \mathbf{X}_{L}^{T} \tilde{\mathbf{y}}_{S I V}^{k}$,

and a non-linear LS estimation for $\boldsymbol{\beta}_{f}$; see e.g. [6], [19][21] for more details.

As in the case of the IDIM-SIV method, the CLOE and the SLS approaches are run without prior data filtering. In both cases, the inertial parameters are initialized with their $\mathrm{CAD}$ values, while the friction parameters are initialized at zero because their values are unknown to the user.

The SLS method converges after 30 iterations to the values given in TABLE 1 which do not match the IDIMSIV estimates. It comes out that The SLS estimates are biased since the IDIM-SIV estimates are consistent. The bias is due to the fact that $\mathbf{X}_{L}(\hat{\mathbf{q}}, \hat{\dot{\mathbf{q}}}, \hat{\ddot{\mathbf{q}}})$ is correlated with $\boldsymbol{\varepsilon}$

. It is worth to note that when associated with the data filtering described in [5], the SLS method converges in 10 iterations to values that are comparable with the IDIMSIV estimates; the variations calculated are less than $2.0 \%$. Unlike the IDM-SIV method and like the standard IDIMLS method, the consistency of the estimates is secured with the SLS approach only if it is associated with proper data filtering.

TABLE 1:

IDIM-SIV, CLOE AND SLS ESTIMATES AFTER CONVERGENCE

\begin{tabular}{|c|c|c|c|}
\hline & $\hat{\boldsymbol{\beta}}_{I V}^{3}$ & $\hat{\boldsymbol{\beta}}_{C L O E}^{50}$ & $\hat{\boldsymbol{\beta}}_{S L S}^{30}$ \\
\hline $\mathrm{ZZ}_{1}$ & $1.25 \mathrm{e} 0(1.3 \mathrm{e}-2)$ & $1.25 \mathrm{e} 0(1.3 \mathrm{e}-2)$ & $6.31 \mathrm{e}-2(3.5 \mathrm{e}-3)$ \\
\hline $\mathrm{Fv}_{1}$ & $11.90 \mathrm{e} 0(0.11 \mathrm{e} 0)$ & $7.00 \mathrm{e} 0(6.3 \mathrm{e}-2)$ & $8.10 \mathrm{e} 0(3.2 \mathrm{e}-2)$ \\
\hline $\mathrm{Fc}_{1}$ & $4.21 \mathrm{e} 0(4.0 \mathrm{e}-2)$ & $6.97 \mathrm{e} 0(1.4 \mathrm{e}-1)$ & $6.06 \mathrm{e} 0(8.4 \mathrm{e}-2)$ \\
\hline $\mathrm{Fs}_{1}$ & $4.78 \mathrm{e} 0(3.80 \mathrm{e}-2)$ & $4.71 \mathrm{e} 0(4.3 \mathrm{e}-2)$ & $4.08 \mathrm{e} 0(3.7 \mathrm{e}-2)$ \\
\hline $\mathrm{Vs}_{1}$ & $2.50 e-2(1.05 e-3)$ & $1.00 \mathrm{e}-2(0.9 \mathrm{e}-3)$ & $2.00 \mathrm{e}-2(9.1 \mathrm{e}-4)$ \\
\hline$\delta_{1}$ & $2.09 \mathrm{e} 0(1.8 \mathrm{e}-2)$ & $2.00 \mathrm{e} 0(2.9 \mathrm{e}-2)$ & $2.51 \mathrm{e} 0(4.9 \mathrm{e}-2)$ \\
\hline $\mathrm{XX}_{2}$ & $-0.48 \mathrm{e} 0(1.5 \mathrm{e}-2)$ & $-0.48 \mathrm{e} 0(1.1 \mathrm{e}-2)$ & $-8.00 e-2(3.3 e-3)$ \\
\hline $\mathrm{XZ}_{2}$ & $-0.16 \mathrm{e} 0(7.8 \mathrm{e}-3)$ & $-0.15 \mathrm{e} 0(5.7 \mathrm{e}-2)$ & $-2.20 \mathrm{e}-2(1.4 \mathrm{e}-3)$ \\
\hline $\mathrm{ZZ}_{2}$ & $1.54 \mathrm{e} 0(1.3 \mathrm{e}-2)$ & $1.54 \mathrm{e} 0(1.3 \mathrm{e}-2)$ & $5.42 \mathrm{e}-2(1.6 \mathrm{e}-3)$ \\
\hline $\mathrm{MX}_{2}$ & $2.84 \mathrm{e} 0(5.4 \mathrm{e}-2)$ & $2.84 \mathrm{e} 0(6.5 \mathrm{e}-2)$ & $4.20 \mathrm{e} 0(2.8 \mathrm{e}-2)$ \\
\hline $\mathrm{Fv}_{2}$ & $8.05 \mathrm{e} 0(8.10 \mathrm{e}-2)$ & $6.15 \mathrm{e} 0(6.8 \mathrm{e}-2)$ & $5.15 \mathrm{e} 0(2.9 \mathrm{e}-2)$ \\
\hline $\mathrm{Fc}_{2}$ & $5.55 \mathrm{e} 0(5.40 \mathrm{e}-2)$ & $7.05 \mathrm{e} 0(9.1 \mathrm{e}-2)$ & $8.26 \mathrm{e} 0(8.1 \mathrm{e}-2)$ \\
\hline $\mathrm{Fs}_{2}$ & $7.97 \mathrm{e} 0(8.54 \mathrm{e}-2)$ & $8.41 \mathrm{e} 0(8.3 \mathrm{e}-2)$ & $7.12 \mathrm{e} 0(7.8 \mathrm{e}-2)$ \\
\hline $\mathrm{Vs}_{2}$ & $5.00 \mathrm{e}-2(2.30 \mathrm{e}-3)$ & $5.00 \mathrm{e}-2(2.9 \mathrm{e}-3)$ & $5.00 \mathrm{e}-2(2.5 \mathrm{e}-3)$ \\
\hline$\delta_{2}$ & $2.78 \mathrm{e} 0(2.53 \mathrm{e}-2)$ & $1.91 \mathrm{e} 0(1.4 \mathrm{e}-2)$ & $2.08 \mathrm{e} 0(1.9 \mathrm{e}-2)$ \\
\hline $\mathrm{XX}_{3}$ & $1.29 \mathrm{e}-1(1.3 \mathrm{e}-2)$ & $0.13 \mathrm{e} 0(1.2 \mathrm{e}-2)$ & $-1.32 \mathrm{e}-2(2.6 \mathrm{e}-3)$ \\
\hline $\mathrm{ZZ}_{3}$ & $1.21 \mathrm{e}-1(8.8 \mathrm{e}-2)$ & $1.12 \mathrm{e}-2(7.1 \mathrm{e}-3)$ & $-5.10 \mathrm{e}-2(1.5 \mathrm{e}-3)$ \\
\hline $\mathrm{MY}_{3}$ & $-6.05 e-2(1.2 e-3)$ & $-6.06 \mathrm{e}-2(1.2 \mathrm{e}-3)$ & $-0.30 \mathrm{e} 0(5.4 \mathrm{e}-2)$ \\
\hline $\mathrm{Ia}_{3}$ & $9.00 e-2(8.1 e-3)$ & $9.87 e-2(9.0 e-3)$ & $5.04 \mathrm{e}-2(1.1 \mathrm{e}-3)$ \\
\hline $\mathrm{Fv}_{3}$ & $4.12 \mathrm{e} 0(4.00 \mathrm{e}-2)$ & $2.00 \mathrm{e} 0(3.0 \mathrm{e}-2)$ & $2.21 \mathrm{e} 0(2.2 \mathrm{e}-2)$ \\
\hline $\mathrm{Fc}_{3}$ & $5.06 \mathrm{e} 0(4.05 \mathrm{e}-2)$ & $5.10 \mathrm{e} 0(3.5 \mathrm{e}-3)$ & $5.56 \mathrm{e} 0(6.1 \mathrm{e}-2)$ \\
\hline $\mathrm{Fs}_{3}$ & $5.81 \mathrm{e} 0(5.72 \mathrm{e}-2)$ & $5.35 \mathrm{e} 0(5.5 \mathrm{e}-2)$ & $5.89 \mathrm{e} 0(5.4 \mathrm{e}-2)$ \\
\hline $\mathrm{Vs}_{3}$ & $5.00 e-2(1.95 e-3)$ & $2.00 \mathrm{e}-2(1.2 \mathrm{e}-3)$ & $2.00 \mathrm{e}-2(1.2 \mathrm{e}-3)$ \\
\hline$\delta_{3}$ & $2.72 \mathrm{e} 0(3.87 \mathrm{e}-2)$ & $2.00 \mathrm{e} 0(4.0 \mathrm{e}-2)$ & $2.70 \mathrm{e} 0(5.4 \mathrm{e}-2)$ \\
\hline $\mathrm{MX}_{4}$ & $-2.10 e-2(4.2 e-3)$ & $-2.90 \mathrm{e}-2(5.3 \mathrm{e}-3)$ & $6.00 \mathrm{e}-2(1.6 \mathrm{e}-3)$ \\
\hline $\mathrm{Ia}_{4}$ & $2.96 e-2(2.7 e-3)$ & $3.12 \mathrm{e}-2(2.9 \mathrm{e}-3)$ & $1.34 \mathrm{e}-2(1.4 \mathrm{e}-3)$ \\
\hline $\mathrm{Fv}_{4}$ & $1.48 \mathrm{e} 0(1.54 \mathrm{e}-2)$ & $1.14 \mathrm{e} 0(1.2 \mathrm{e}-2)$ & $1.20 \mathrm{e} 0(2.3 \mathrm{e}-2)$ \\
\hline $\mathrm{Fc}_{4}$ & $2.07 \mathrm{e} 0(2.00 \mathrm{e}-2)$ & $2.00 \mathrm{e} 0(5.7 \mathrm{e}-2)$ & $2.30 \mathrm{e} 0(7.8 \mathrm{e}-2)$ \\
\hline $\mathrm{Fs}_{4}$ & $2.15 \mathrm{e} 0(1.84 \mathrm{e}-2)$ & $1.95 \mathrm{e} 0(1.8 \mathrm{e}-2)$ & $2.35 \mathrm{e} 0(1.5 \mathrm{e}-2)$ \\
\hline $\mathrm{Vs}_{4}$ & $5.00 \mathrm{e}-2(2.40 \mathrm{e}-3)$ & $5.00 \mathrm{e}-2(2.5 \mathrm{e}-3)$ & $5.00 \mathrm{e}-2(2.5 \mathrm{e}-3)$ \\
\hline$\delta_{4}$ & $1.60 \mathrm{e} 0(3.81 \mathrm{e}-2)$ & $1.50 \mathrm{e} 0(3.7 \mathrm{e}-2)$ & $1.00 \mathrm{e} 0(4.1 \mathrm{e}-2)$ \\
\hline $\mathrm{MY}_{5}$ & $-3.0 \mathrm{e}-2(4.2 \mathrm{e}-3)$ & $-4.00 e-3(5.9 e-4)$ & $-2.30 e-2(1.7 e-3)$ \\
\hline $\mathrm{Ia}_{5}$ & $4.10 \mathrm{e}-2(4.4 \mathrm{e}-3)$ & $4.10 e-3(3.7 e-4)$ & $1.00 \mathrm{e}-2(6.8 \mathrm{e}-3)$ \\
\hline $\mathrm{Fv}_{5}$ & $1.90 \mathrm{e} 0(3.8 \mathrm{e}-2)$ & $1.85 \mathrm{e} 0(2.1 \mathrm{e}-2)$ & $1.84 \mathrm{e} 0(3.7 \mathrm{e}-2)$ \\
\hline $\mathrm{Fc}_{5}$ & $2.80 \mathrm{e} 0(6.4 \mathrm{e}-2)$ & $2.90 \mathrm{e} 0(1.2 \mathrm{e}-1)$ & $2.85 \mathrm{e} 0(4.5 \mathrm{e}-2)$ \\
\hline $\mathrm{Ia}_{6}$ & $9.85 \mathrm{e}-2(9.4 \mathrm{e}-3)$ & $0.99 \mathrm{e}-2(9.5 \mathrm{e}-4)$ & $1.23 \mathrm{e}-3(2.4 \mathrm{e}-4)$ \\
\hline $\mathrm{Fv}_{6}$ & $0.69 \mathrm{e} 0(1.4 \mathrm{e}-2)$ & $0.45 \mathrm{e} 0(5.4 \mathrm{e}-3)$ & $0.68 \mathrm{e} 0(1.4 \mathrm{e}-3)$ \\
\hline $\mathrm{Fc}_{6}$ & $2.00 \mathrm{e} 0(6.1 \mathrm{e}-2)$ & $2.20 \mathrm{e} 0(6.4 \mathrm{e}-2)$ & $2.00 \mathrm{e} 0(7.6 \mathrm{e}-2)$ \\
\hline
\end{tabular}

The standard CLOE method converges after 50 iterations to the values given in TABLE 1. The friction parameters are not well identified by this approach since they differ significantly to the IDIM-SIV estimates. For instance, $F c_{j}$ is greater than $F s_{j}$ for the first and fourth joints which is physically impossible. This result is 
explained by the fact that $\mathbf{q}_{S}$ is not sensitive enough to parameters' variations because of the controller yielding a small tracking error. The following approximation, $q_{j} \approx q_{r_{j}}$, holds. It comes out that the jacobian matrix of $J(\boldsymbol{\beta})$, denoted as $\mathbf{X}_{\delta}$, has small singular values; the maximal (resp. minimal) singular value equals $2.4 \mathrm{e}-3$ (resp. 1.5e-6). These small singular values can be compared with the singular values of $\mathbf{X}$, where the greatest and smallest values are 350 and 0.6, respectively. There is thus little information in $\mathbf{X}_{\delta}$, and this explains why the usual CLOE method converges slowly and is very sensitive to initialization.

\section{CONCLUSION}

This paper has introduced a new approach to robot identification called the IDIM-SIV method. This method consists of two main steps. In the first of these, the estimation of the significant nonlinearities in the system is combined with the inverse dynamic model to identify the graphical nature of the friction characteristics. In the second step, the inertia and gravity parameters are estimated by an iterative IV approach based on the inverse dynamic model and the estimated friction characteristics.

Finally, this new two-step IDIM-SIV identification procedure has been compared thoroughly with a standard Closed-Loop Output-Error method and the Separable Least Squares technique. The results show that IDIM-SIV outperforms these approaches in terms of iterations required to converge, the accuracy of estimates, and robustness to noise in the absence of data filtering.

\section{ACKNOWLEDGMENT}

The author is grateful to Philippe Lemoine for developing the software and running the experiments. $\mathrm{He}$ is also grateful to Maxime Gautier for providing the data.

\section{REFERENCES}

[1] K. Kozlowski. "Modelling and Identification in Robotics," Springer Verlag London Limited, Great Britain, 1998.

[2] J. Swevers, W. Verdonck, and J. De Schutter, "Dynamic model identification for industrial robots - Integrated experiment design and parameter estimation," IEEE Control Systems Magazine, vol. 27, 2007, pp. 58-71.

[3] J. Wu, J. Wang and Z. You "An overview of dynamic parameter identification of robots," Robotics and Computer-Integrated Manufacturing, Vol. 26(5), October 2010, pp. 414-419.

[4] A. Calanca, L. M. Capisani, A. Ferrara, and L. Magnani, "MIMO Closed Loop Identification of an Industrial Robot," IEEE Transactions on Control System Technology, Vol. 19(5), September 2011, pp. 1214-1224.

[5] M. Gautier, A. Janot, and P.O. Vandanjon, "A New Closed-Loop Output Error Method for Parameter Identification of Robot Dynamics," IEEE Transactions on Control System Technology, Vol. 21(2), 2013, pp.428-444.

[6] A. Janot, P.O. Vandanjon and M. Gautier, "A Generic Instrumental Variable Approach for Industrial Robots Identification," IEEE Transactions on Control Systems Technology, Vol. 22(1), pp. 132145.

[7] L. Simoni, M. Beschi, G. Legnani, and A. Visioli, "Modelling the temperature in joint friction of industrial manipulators," Robotica, Vol. 37(5), May 2019, pp. 906-927.

[8] C. Gaz, M. Cognetti, A. Oliva, P. R. Giordano and A. De Luca, "Dynamic Identification of the Franka Emika Panda Robot With
Retrieval of Feasible Parameters Using Penalty-Based Optimization," IEEE Robotics and Automation Letters, Vol. 4(4), October 2019, pp. 4147 - 4154.

[9] T. Lee, P. M. Wensing and F. C. Park, "Geometric Robot Dynamic Identification: A Convex Programming Approach," IEEE Transactions on Robotics, Vol. 36(2), April 2020, 348 - 365.

[10] M. Indri and S. Trapani, "Framework for Static and Dynamic Friction Identification for Industrial Manipulators," IEEE/ASME Transactions on Mechatronics, Vol. 25(3), June 2020, pp. 15891599.

[11] Y. Han, J. Wu, C. Liu and Z. Xiong, "An Iterative Approach for Accurate Dynamic Model Identification of Industrial Robots," IEEE Transactions on Robotics, Early access, May 2020, pp. 1-18.

[12] H. Garnier and L. Wang (Eds), "Identification of Continuous-time Models from Sampled Data," Springer, 2008.

[13] Gilson M., Garnier H., Young P.C. and Van den Hof P. (2011), "Optimal instrumental variable method for closed-loop identification", Control Theory \& Applications, IET, vol. 5, no. 10, pp. $1147-1154$.

[14] P.C. Young, "Recursive Estimation and Time-Series Analysis: An Introduction for the Student and Practitioner," Springer Verlag, 2nd Edition., 2011.

[15] A. Janot, P.O. Vandanjon and M. Gautier, "A Durbin-Wu-Hausman Test for Industrial Robot Identification," Control Engineering Practice, Vol. 48, pp. 52-62.

[16] B. Bona and M. Indri, "Friction compensation in robotics: An overview," In: Proc. 44th Decision Control Conf., Eur. Control Conf., Seville, Spain, 2005, pp. 4360-4367.

[17] A. C. Bittencourt and P. Axelsson, "Modeling and experiment design for identification of wear in a robot joint under load and temperature uncertainties based on friction data," IEEE/ASME Transactions on Mechatronics, vol. 19, no. 5, pp. 1694-1706, 2014.

[18] AC, Bittencourt and S. Gunnarsson, "Static friction in a robot joint-modeling and identification of load and temperature effects." J Dynam Syst Meas Control, 2012;134(5).

[19] G. H. Golub and V. Pereyra, "The Differentiation of PseudoInverses and Nonlinear Least Squares Problems Whose Variables Separate," SIAM Journal on Numerical Analysis, Vol. 10, No. 2 (Apr., 1973), pp. 413-432.

[20] Previdi, F. \& Lovera, M. (2004). Identification of non-linear parametrically varying models using separable least squares. International Journal of Control, 2004, 77, pp. 1382-1392.

[21] S.M. Hashemi and H. Werner, "Parameter Identification of a Robot Arm Using Separable Least Squares Technique," In: Proceedings of 10th European Control Conference, Budapest, Hungary, August 2009.

[22] W. Khalil and E. Dombre, "Modeling, identification and control of robots", Hermes Penton, London, 2002.

[23] Briot S. and Gautier M., "Global Identification of Joint Drive Gains and Dynamic Parameters of Parallel Robots," Multibody System Dynamics, DOI 10.1007/s11044-013-9403-6, in press

[24] R. Davidson and J.G. MacKinnon, "Estimation and Inference in Econometrics," Oxford University Press, New York, 1993.

[25] M. Gautier and W. Khalil, "Exciting trajectories for the identification of the inertial parameters of robots," International Journal of Robotics Research, vol. 11, Aug. 1992, pp. 362-375.

[26] E. Walter and L. Pronzato, Identification of parametric models from experimental data. Springer, 1997. 